\title{
Investigação do Processo de Absorção de Água de Hidrogéis de Polissacarídeo: Efeito da Carga lônica, Presença de Sais, Concentrações de Monômero e Polissacarídeo
}

\author{
Adriel Bortolin \\ Laboratório Nacional de Nanotecnologia para o Agronegócio, Embrapa Instrumentação, São Carlos \\ Departamento de Química, UFSCar \\ Fauze A. Aouada \\ Laboratório Nacional de Nanotecnologia para o Agronegócio, Embrapa Instrumentação, São Carlos \\ Instituto de Química, UNESP \\ Elson Longo \\ Instituto de Química, UNESP \\ Luiz H. C. Mattoso \\ Laboratório Nacional de Nanotecnologia para o Agronegócio, Embrapa Instrumentação, São Carlos
}

\begin{abstract}
Resumo: Neste trabalho foi reportada a caracterização de hidrogéis constituídos por metilcelulose (MC) e poliacrilamida (PAAm) preparados pelo processo de reticulação cruzada. As propriedades espectroscópicas e morfológicas foram investigadas por espectroscopia no infravermelho com transformada de Fourier e microscopia eletrônica de varredura, respectivamente. O efeito da carga iônica, presença de sais, concentrações de monômero AAm e polissacarídeo MC na absorção de água e nas propriedades cinéticas dos hidrogéis foi detalhadamente investigado. Os resultados indicaram que o decréscimo da concentração de MC ou aumento da concentração de AAm, carga iônica do contra-íon do sal de cloreto e a presença de fertilizante no meio externo de intumescimento provocaram diminuição significativa na absorção de água dos hidrogéis. O mecanismo de absorção de água dos hidrogéis de PAAm-MC em água seguiu o modelo de difusão Fickiana; já o mecanismo dos hidrogéis intumescidos em sais (cloreto ou fertilizante) seguiu o modelo de transporte anômalo. Pela alta e rápida absorção de água, as matrizes porosas e tridimensionais compostas por PAAm e MC podem em potencial ser aplicadas na agricultura como veículos carreadores.
\end{abstract}

Palavras-chave: Absorção de água, difusão Fickiana, hidrogéis biodegradáveis, propriedades cinéticas, propriedades morfológicas.

\section{Investigation of Water Absorption Process in Polysaccharide Hydrogels: Effect of Ionic Charge, Presence of Salt, Monomer and Polysaccharide Concentrations}

Abstract: This work reported the characterization of hydrogels constituted by methylcellulose (MC) entrapped in polyacrylamide (PAAm) network prepared by cross-linking polymerization (gelling process). Spectroscopic and morphologic properties were investigated using Fourier transform infrared spectroscopy and scanning electron microscopy, respectively. Effects of AAm, MC contents, ionic charge of salt counter-ions $\left(\mathrm{NaCl}, \mathrm{KCl}, \mathrm{NH}_{4} \mathrm{Cl}, \mathrm{CaCl}_{2}, \mathrm{MnCl}_{2}, \mathrm{MgCl}_{2}\right.$, and $\left.\mathrm{AlCl}_{3}\right)$, and fertilizers $\left[\left(\mathrm{NH}_{4}\right)_{2} \mathrm{SO}_{4}\right.$ and $\left.\mathrm{KH}_{2} \mathrm{PO}_{4}\right]$ on kinetic and hydrophilic (swelling degree) properties were investigated in detail. By decreasing the $\mathrm{MC}$ content or by increasing acrylamide content, there was a pronounced decrease in water uptake by the hydrogels. The increase in counter-ion X-Cl salt and the presence of fertilizer on the external swelling medium also provoked a decrease in water uptake. The water uptake mechanism of PAAm-MC hydrogels swollen in water followed the Fickian diffusion, while the mechanisms of hydrogels swollen in salt or fertilizer aqueous media followed the anomalous transport process. With their fast and high water absorption, the porous, three-dimensional matrices containing PAAm and MC are promising for agriculture as carrier vehicles.

Keywords: Water absorption, Fickian diffusion, biodegradable hydrogels, kinetic properties, morphologic properties.

\section{Introdução}

Os hidrogéis são materiais poliméricos constituídos por uma ou mais redes tridimensionalmente estruturadas, formadas por cadeias macromoleculares interligadas por ligações covalentes (reticulações) ou interações físicas ${ }^{[1]}$ que em condições específicas podem absorver grande quantidade de água ${ }^{[2,3]}$. Essa propriedade os qualifica como componentes importantes em processos industriais, particularmente na produção de materiais de uso médico $^{[4-6]}$ e agrícola ${ }^{[7,8]}$.
Os hidrogéis podem ser sintetizados a partir de materiais naturais ou sintéticos. Hidrogéis preparados a partir de polímeros naturais podem apresentar propriedades mecânicas não satisfatórias e fornecem substratos para o desenvolvimento de patógenos que provocam respostas inflamatórias. Por outro lado se destacam pela biocompatibilidade, biodegradabilidade e reconhecimento de moléculas biológicas ${ }^{[9]}$. Alguns hidrogéis sintéticos não apresentam propriedades bioativas, fato que restringe sua aplicação na área 
médica, porém apresentam excelentes propriedades mecânicas e hidrofílicas ${ }^{[10]}$. Os hidrogéis sintetizados a partir da combinação de polímeros sintéticos (por exemplo, poliacrilamida - PAAm) e naturais vêm sendo desenvolvidos de forma estratégica de modo a otimizar os processos mecânicos e biológicos, ampliando sua aplicação nas áreas médicas e tecnológicas.

O polissacarídeo metilcelulose (MC) é um polímero polihidroxilado hidrofílico solúvel em meio aquoso que pode ser transformado em gel através de reticulação química utilizando di-aldeídos na presença de ácido forte ${ }^{[11]}$. No entanto, apresentam pobre resistência mecânica, limitando sua aplicação tecnológica. Essa limitação pode ser minimizada quando o mesmo é suportado mecanicamente em redes poliméricas constituídas por polímeros sintéticos, por exemplo, PAAm.

Neste trabalho foi investigado o efeito da carga iônica, presença de sais, concentrações de monômero acrilamida e polissacarídeo metilcelulose na absorção de água e nas propriedades cinéticas [expoente difusional $(n)$ e constante de difusão $(k)$ ] de hidrogéis de poliacrilamida e metilcelulose. Adicionalmente, as propriedades espectroscópicas e morfológicas dos hidrogéis foram analisadas por meio da espectroscopia no infravermelho com transformada de Fourier (FTIR) e microscopia eletrônica de varredura (MEV), respectivamente.

\section{Experimental}

\section{Síntese dos hidrogéis}

Os hidrogéis constituídos de poliacrilamida (PAAm) e do polissacarídeo biodegradável metilcelulose (MC, $\mathrm{Mn}=40,000 \mathrm{~g} . \mathrm{mol}^{-1}$, viscosidade $400 \mathrm{cP}$, com 27,5-31,5\% de grupamentos metila, 68,5-72,5\% de grupamentos hidroxila, dados fornecidos pelo fabricante Aldrich) foram sintetizados de acordo com o procedimento recentemente publicado pelo nosso grupo de pesquisa ${ }^{[12,13]}$. Os hidrogéis foram obtidos por meio de polimerização química do monômero acrilamida (AAm, Fluka), em solução aquosa contendo MC e do agente de reticulação N'-N-metilenobisacrilamida (MBAAm, Aldrich) utilizando N,N,N',N'-tetrametil-etilenobisacrilamida (TEMED, Sigma) como catalisador. A concentração de MBAAm foi fixada em 2 mol\% em relação a concentração de AAm. Persulfato de sódio $\left(\mathrm{Na}_{2} \mathrm{~S}_{2} \mathrm{O}_{8}\right.$, Sigma) foi adicionado com intuito de iniciar a reação de polimerização via radical livre ${ }^{[14]}$. A solubilização dos reagentes e a síntese dos hidrogéis foram realizadas em temperatura ambiente.

\section{Grau de intumescimento (Q)}

A capacidade de absorção de água pelos hidrogéis foi observada por medidas de grau de intumescimento (Q) em função do tempo. Os estudos de intumescimento foram realizados em água destilada/deionizada (controle) e soluções aquosas dos fertilizantes sulfato de amônio e fosfato de potássio a aproximadamente $1900 \mathrm{mg} \mathrm{L}^{-1}$. Para verificar o efeito da carga iônica presente nos contra-íons de sais $\mathrm{X}-\mathrm{Cl}$ (onde $\mathrm{X}$ representa o contra-íon), sete diferentes meios salinos a $0,15 \mathrm{~mol} \mathrm{~L}^{-1}$ e $25,0{ }^{\circ} \mathrm{C}$ foram utilizados: íons monovalentes $\left(\mathrm{NaCl}, \mathrm{KCl}, \mathrm{NH}_{4} \mathrm{Cl}\right)$, divalentes $\left(\mathrm{CaCl}_{2}, \mathrm{MnCl}_{2}\right.$, $\left.\mathrm{MgCl}_{2}\right)$ e trivalente $\left(\mathrm{AlCl}_{3}\right)$.

Depois de purificados, os hidrogéis foram cortados em forma circular, e secos em estufa com temperatura controlada de $30,0{ }^{\circ} \mathrm{C}$. Para as determinações de $\mathrm{Q}$, os hidrogéis desidratados foram pesados em balança analítica e submetidos ao intumescimento nos meios propostos. As medidas de grau de intumescimento foram realizadas em intervalos de tempo " $t$ " pré-determinados, sendo que em cada tempo " $t$ " o hidrogel era retirado da solução de intumescimento e cuidadosamente pesado. Os valores de $\mathrm{Q}$ foram obtidos por meio de razão da massa dos hidrogéis intumescidos e a massa dos hidrogéis $\operatorname{secos}{ }^{[15]}$. As medidas foram realizadas em triplicata e as barras de erros correspondem ao desvio padrão $(\sigma)$ (média $\pm \sigma, \mathrm{n}=3$ ).

\section{Parâmetros cinéticos dos hidrogéis}

Os parâmetros da cinética de intumescimento [expoente difusional $(n)$ e constante de difusão $(k)$ ] foram calculados utilizando a Equação $1^{[16]}$ :

$$
\frac{M_{t}}{M_{e q}}=k t^{n}
$$

onde t é o tempo, $k$ é a constante de difusão e depende do tipo do hidrogel e do meio de intumescimento, $n$ é o expoente difusional, que fornece informação sobre o tipo de mecanismo de transporte que impulsiona a liberação/sorção de um dado soluto. $M_{t}$ e $M_{e q}$ são as massas do hidrogel em um tempo "t" de intumescimento e no estado de equilíbrio, respectivamente.

\section{Espectroscopia no infravermelho com transformada de Fourier (FTIR)}

Os espectros dos hidrogéis de PAAm-MC foram obtidos utilizando um espectrômetro Perkin Elmer Spectrum (modelo Paragon 1000). Os hidrogéis sintetizados foram retirados do meio de intumescimento, liofilizados, misturados com brometo de potássio $(\mathrm{KBr})$ e prensados em alta pressão ${ }^{[17]}$. Espectros de FTIR foram obtidos registrando 128 varreduras de 400 a $4000 \mathrm{~cm}^{-1}$, com resolução de $2 \mathrm{~cm}^{-1}$.

\section{Microscopia eletrônica de varredura (MEV)}

Depois de intumescidos até equilíbrio em água, os hidrogéis foram retirados e imersos em nitrogênio líquido. Posteriormente, as amostras foram liofilizadas por aproximadamente 24 horas. As amostras de hidrogéis foram depositadas em um porta amostra e suas superfícies foram recobertas com uma fina camada de ouro ${ }^{[18]}$. Com esse procedimento, a estrutura porosa do hidrogel não é colapsada, assegurando assim que todas as características morfológicas obtidas para os hidrogéis secos podem ser utilizadas para os hidrogéis no estado intumescido ${ }^{[19]}$. As micrografias dos hidrogéis foram obtidas utilizando um microscópio eletrônico de varredura Shimadzu, modelo Hitachi S-570.

\section{Resultados e Discussão}

\section{Grau de intumescimento (Q)}

Quando um hidrogel é colocado em água ou outro meio de intumescimento, suas cadeias poliméricas podem interagir com o meio, fazendo com que elas se expandam. Quando as redes não mais se expandirem, o estado de equilíbrio do hidrogel é atingido, ou seja, é o instante em que não há mais variações gravimétricas significativas (variações de massa atribuídas à absorção de água) ${ }^{[20]}$.

O grau de intumescimento é um parâmetro muito importante para várias aplicações dos hidrogéis ${ }^{[2]}$. Neste trabalho, a influência dos efeitos das concentrações de acrilamida e metilcelulose, carga iônica e presença dos fertilizante sulfato de amônio e fosfato de potássio na absorção de água dos hidrogéis de PAAm-MC foi avaliada por medidas de grau de intumescimento. Os resultados serão discutidos a seguir. 


\section{Efeito das concentrações de acrilamida e metilcelulose}

Foram sintetizados hidrogéis a partir de três concentrações diferentes de acrilamida: 6,0; 9,0 e 12,0\% (m/v). Para cada concentração de acrilamida foram estudadas duas concentrações de metilcelulose: 0,5 e $1,0 \%(\mathrm{~m} / \mathrm{v})$. A hidrofilicidade dos hidrogéis foi verificada por meio de curvas de grau de intumescimento (Q) em função do tempo. Para obtenção dos valores de $\mathrm{Q}$ no equilíbrio $\left(\mathrm{Q}_{\mathrm{eq}}\right)$, foi tomada como base a variação da massa do hidrogel, por um período de 24 horas.

As dependências do grau de intumescimento em função do tempo para hidrogéis sintetizados com $6,0 \%$ de AAm com diferentes quantidades de MC são mostradas na Figura 1. Na Figura 2 são mostrados os valores do grau de intumescimento no estado de equilíbrio $\left(\mathrm{Q}_{\mathrm{eq}}\right)$ para todos os hidrogéis estudados. Ambas as Figuras foram obtidas utilizando água destilada como meio externo de intumescimento. Após o hidrogel atingir o estado de equilíbrio de intumescimento, os valores de $\mathrm{Q}_{\mathrm{eq}}$ praticamente não apresentaram mais variação em função do tempo até 50 horas de estudo. Isso é um bom indicativo da preservação da estabilidade química dos hidrogéis. Além disso, a não variação da absorção de água após o estado de equilíbrio, também é um indicativo que os grupamentos amida não foram hidrolisados, ou seja, não se converteram a

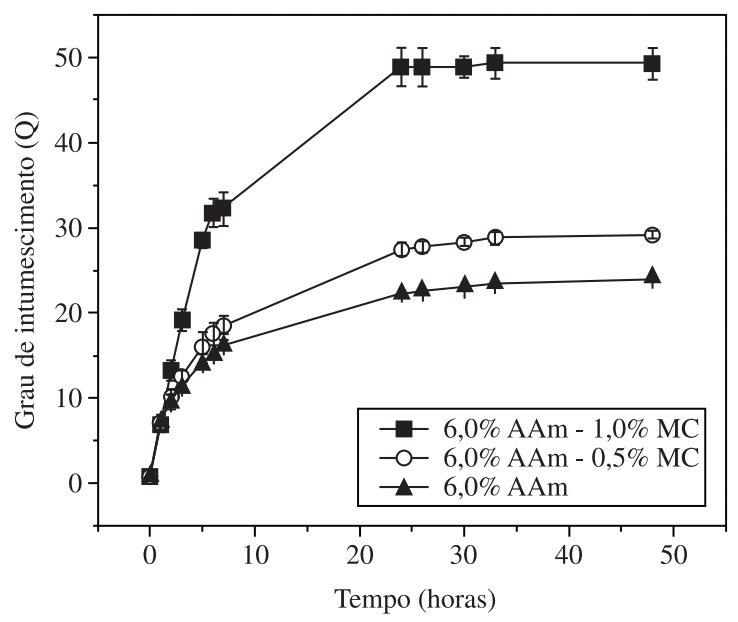

Figura 1. Dependência do grau de intumescimento $(\mathrm{Q})$ em função do tempo para hidrogéis de PAAm-MC com diferentes composições a $25,0 \pm 0,1^{\circ} \mathrm{C}$.

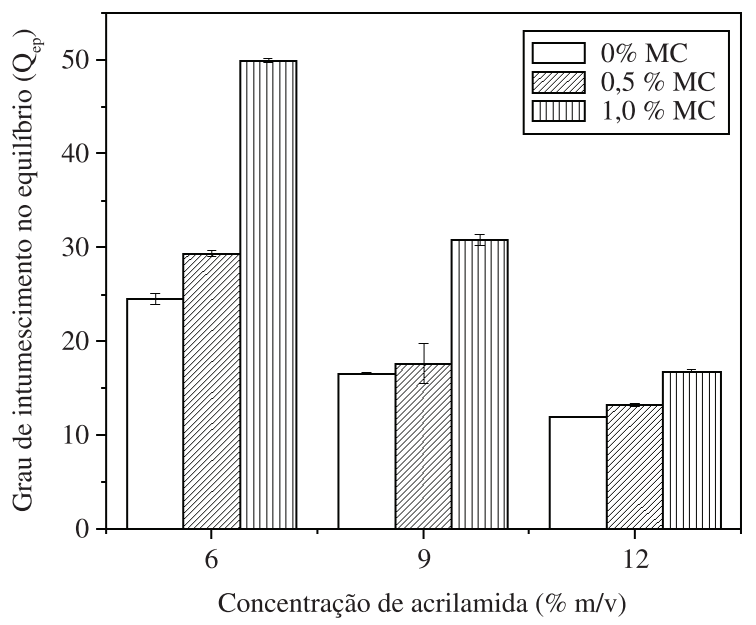

Figura 2. Dependência dos valores de grau de intumescimento no equilíbrio $\left(Q_{e q}\right)$ em função da concentração de acrilamida para os hidrogéis de PAAm-MC com diferentes composições a $25,0 \pm 0,1{ }^{\circ} \mathrm{C}$. grupamentos carboxílicos $\left(-\mathrm{CONH}_{2}+\mathrm{H}_{2} \mathrm{O} \rightarrow-\mathrm{COOH}+\mathrm{NH}_{3}\right)$ ao final do experimento.

Para uma quantidade fixa de $\mathrm{MC}$ os valores de $\mathrm{Q}_{\mathrm{eq}}$ diminuíram significativamente com o aumento da concentração de AAm devido ao aumento na rigidez das cadeias poliméricas, provocando uma menor capacidade de absorção de água. De maneira geral, redes poliméricas formadas com alta concentração de AAm são mais fortemente reticuladas e isso reflete em uma menor expansão e o volume de água que difunde para o interior da matriz é menor ${ }^{[21]}$. Resultados de propriedades mecânicas obtidos em estudos anteriores $^{[22]}$ neste mesmo tipo de hidrogel comprovaram que o aumento da rigidez da matriz de hidrogel é estritamente relacionada com o aumento da concentração de AAm e/ou diminuição da concentração de MC.

Da mesma forma, para teores equivalentes de AAm, os valores de $\mathrm{Q}_{\text {eq }}$ aumentam a medida em que a concentração de $\mathrm{MC}$ é aumentada. Isso está relacionado à incorporação de grupamentos hidroxila provenientes da $\mathrm{MC}$, que aumentam a hidrofilicidade dos hidrogéis.

\section{Efeito da carga iônica}

Os valores obtidos de $\mathrm{Q}_{\mathrm{eq}}$ em função da solução salina de intumescimento são expressos na Figura 3. Foi verificado que os mesmos reduziram drasticamente quando o meio de intumescimento foi variado de água para solução contendo íons. Resultados similares para outros sistemas de hidrogéis foram discutidos nos trabalhos desenvolvidos por Liang et al. (2004) ${ }^{[23]} \mathrm{e}$ Liang et al. (2009) ${ }^{[24]}$. Quando o hidrogel é imerso em uma solução contendo íons positivos, podem ocorrer interações localizadas entre os grupamentos hidroxila (presentes nas cadeias do polissacarídeo $\mathrm{MC}$ ) com os contra-íons $\mathrm{X}^{\mathrm{n}+}$ do sal $\mathrm{X}-\mathrm{Cl}$, o que pode induzir a formação de pares iônicos (ou complexos iônicos) - O--- $\mathrm{X}^{\mathrm{n}+}$ - entre as espécies. Consequentemente, possíveis repulsões eletrostáticas entre os segmentos das cadeias de MC, na qual se encontram entrelaçadas nas cadeias de PAAm reticuladas, são minimizadas dificultando as expansões localizadas das redes dos hidrogéis. Além disso, em condições extremas pode ocorrer até a contração das redes dos hidrogéis, o que contribui para menor absorção de água do hidrogel devido à diminuição da afinidade das mesmas com moléculas de água.

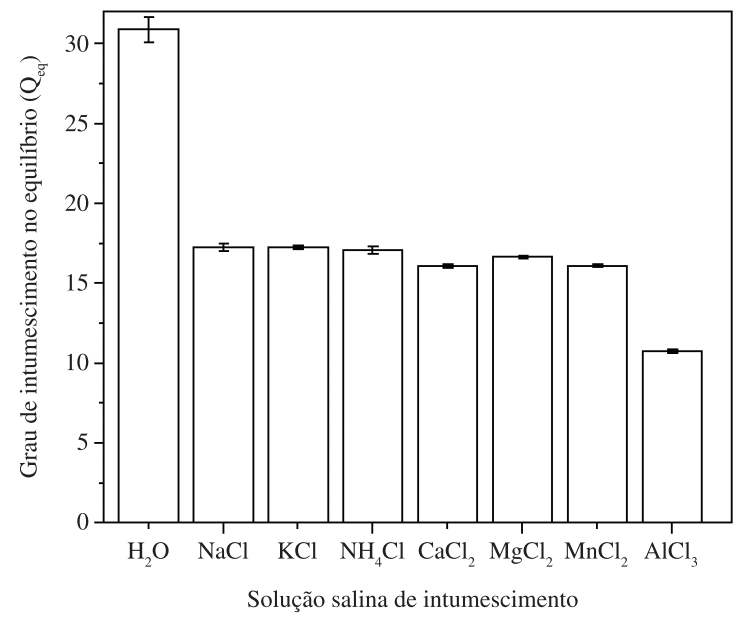

Figura 3. Dependência dos valores de grau de intumescimento no equilíbrio $\left(Q_{e q}\right)$ do hidrogel composto por $9,0 \%$ AAm e 1,0\% MC em função da solução salina de intumescimento a $0,15 \mathrm{~mol} \mathrm{~L}^{-1}$ e $25,0{ }^{\circ} \mathrm{C}$ : íons monovalentes $\left(\mathrm{NaCl}, \mathrm{KCl}, \mathrm{NH}_{4} \mathrm{Cl}\right)$, divalentes $\left(\mathrm{CaCl}_{2}, \mathrm{MnCl}_{2}, \mathrm{MgCl}_{2}\right)$ e trivalente $\left(\mathrm{AlCl}_{3}\right)$. 
Adicionalmente, a diminuição de $\mathrm{Q}_{\mathrm{eq}}$ foi mais acentuada para a solução contendo íons $\mathrm{Al}^{3+}$, como era esperado. As interações entre os grupamentos hidroxila e os contra-íons $\mathrm{X}^{\mathrm{n}+}$ se dão mais fortemente à medida que a valência do contra-íon aumenta, ou seja: $-\mathrm{O}---\mathrm{X}^{+}-<-(\mathrm{O})_{2}---\mathrm{X}^{2+}-<-(\mathrm{O})_{3}---\mathrm{X}^{3+}-$. Além disso, os cátions trivalentes possuem uma alta densidade de carga positiva; o que potencializa sua interação podendo até formar ligações eletrostáticas intra- e inter-moleculares com os segmentos poliméricos formadores das redes dos hidrogéis. Como conseqüência direta, pontos de reticulações físicas e/ou químicas poderão se formar contribuindo para o aumento da densidade de reticulação dos hidrogéis. De maneira geral, redes de hidrogéis mais fortemente reticuladas absorvem menos água devido à sua pequena flexibilidade.

\section{Efeito dos fertilizantes sulfato de amônio e fosfato de potássio}

A capacidade de intumescimento dos hidrogéis em solução de fertilizantes (sulfato de amônio $\left(\mathrm{NH}_{4}\right)_{2} \mathrm{SO}_{4}$ e fosfato de potássio $\left(\mathrm{KH}_{2} \mathrm{PO}_{4}\right)$ estão apresentados na Figura 4. A presença do fertilizante no meio de intumescimento diminuiu o grau de intumescimento dos hidrogéis devido às interações dos grupamentos iônicos $\mathrm{NH}_{4}^{+} \mathrm{e} \mathrm{K}^{+}$ oriundos dos fertilizantes com os grupamentos hidroxila oriundo da $\mathrm{MC}$ e grupamentos $\mathrm{SO}_{4}{ }^{2-}, \mathrm{H}_{2} \mathrm{PO}_{4}^{-}$e $\mathrm{PO}_{4}{ }^{2-}-$ segmentos $\mathrm{CONH}_{2}$

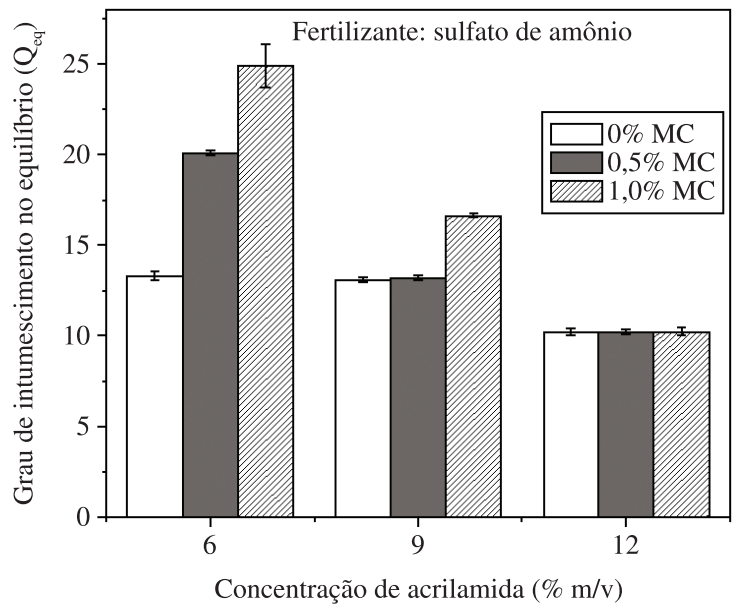

(a)

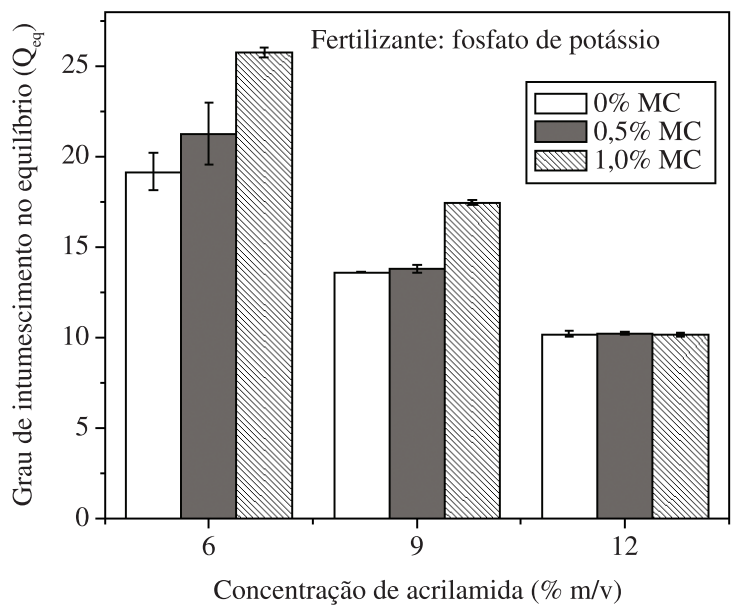

(b)

Figura 4. Dependência dos valores de grau de intumescimento no equilíbrio $\left(Q_{\text {eq }}\right)$ em função da concentração de acrilamida para os hidrogéis de PAAm-MC com diferentes composições a $25,0 \pm 0,1{ }^{\circ} \mathrm{C}$ : (a) sulfato de amônio e (b) fosfato de potássio. presentes nas cadeias poliméricas de PAAm e MBAAm. Essas interações diminuem também a expansão das redes tridimensionais porosas que formam os hidrogéis. Xu et al. (2011) ${ }^{[25]}$ constataram a formação de um complexo de coordenação entre ânions fosfato com grupos aminas do tipo $\mathrm{R}-\mathrm{N}+\left(\mathrm{CH}_{2} \mathrm{CH}_{3}\right)_{3} \ldots \ldots \ldots . . \mathrm{H}_{2} \mathrm{PO}_{4}^{-}$por meio da variação espectral FTIR da banda em aproximadamente $1345 \mathrm{~cm}^{-1}$. Baseando nessas informações da literatura, as interações entre os grupos $\mathrm{SO}_{4}^{-}, \mathrm{PO}_{4}^{-}$e $\mathrm{H}_{2} \mathrm{PO}_{4}^{-}$com os grupos $-\mathrm{CONH}_{2}$ possivelmente ocorrem através de formação de complexos similares, tais como: $-\mathrm{CONH}_{2}+\ldots \ldots . . . \mathrm{SO}_{4}^{-} ;-\mathrm{CONH}_{2}+\ldots \ldots . . . \mathrm{PO}_{4}^{-} \mathrm{e}-\mathrm{CONH}_{2}+\ldots \ldots \ldots \mathrm{H}_{2} \mathrm{PO}_{4}^{-}$. Adicionalmente, uma possível formação do complexo dos ânions fosfato foi observada pela formação de uma banda FTIR em aproximadamente $1348 \mathrm{~cm}^{-1}$ (ver Figura 5b).

$\mathrm{O}$ aumento da concentração do polissacarídeo MC provoca maior absorção de água (maior grau de intumescimento) para ambos os fertilizantes. Porém é observado na Figura 4, para hidrogéis contendo $12,0 \%$ de AAm, a variação da concentração de metilcelulose praticamente não afetou o grau de intumescimento. Isto pode ser atribuído a menor mobilidade dos grupamentos hidroxila que estão entrelaçados em redes poliméricas tridimensionais de PAAm altamente rígidas ${ }^{[21]}$, somada a interação destes grupamentos com os íons presentes na solução de intumescimento.

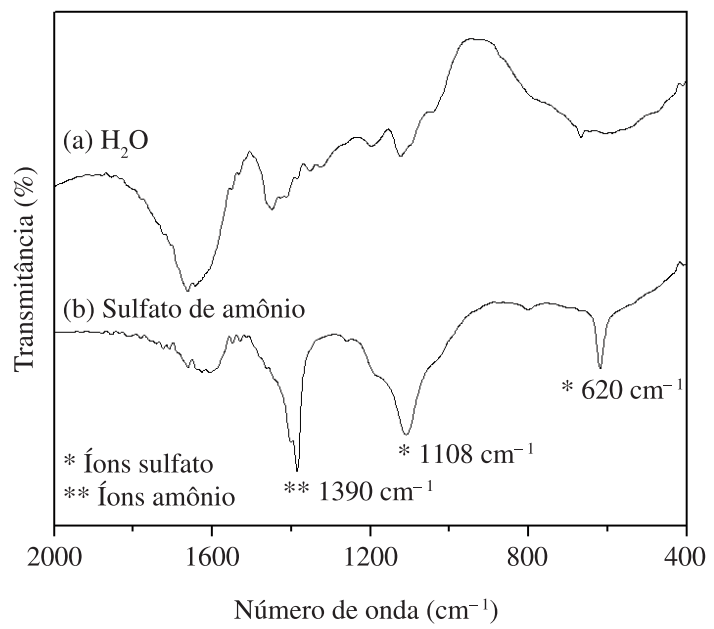

(a)

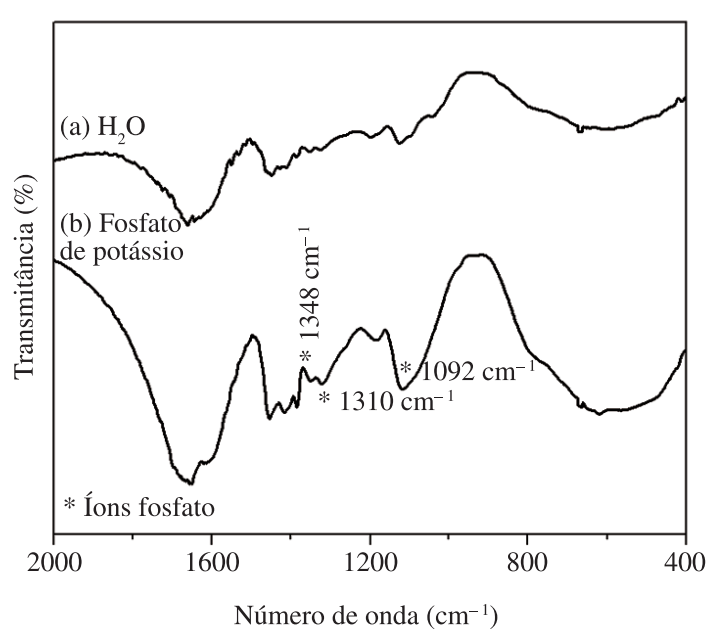

(b)

Figura 5. Espectros de FTIR dos hidrogéis de PAAm-MC compostos por 6,0\% AAm e 1,0\% MC intumescidos em água e PAAm com (a) sulfato de amônio $\left(\mathrm{NH}_{4}\right)_{2} \mathrm{SO}_{4} \mathrm{e}$ (b) fosfato de potássio $\left(\mathrm{KH}_{2} \mathrm{PO}_{4}\right)$ incorporados. 


\section{Espectroscopia no infravermelho com transformada de Fourier (FTIR)}

A Figura 5 apresenta espectros de FTIR para um mesmo tipo de hidrogel, sintetizado com 6,0\% AAm e 1,0\% de MC intumescidos em água e em solução de fertilizantes (a) fosfato de potássio e (b) sulfato de amônio. A incorporação de fosfato de potássio foi identificada pelas bandas na região de $1310 \mathrm{~cm}^{-1}$, na qual é característica de grupamentos $\mathrm{P}=\mathrm{O}$ e também na região de $1092 \mathrm{~cm}^{-1}$ característica de íons fosfato ${ }^{[26]}$. No hidrogel intumescido em solução de sulfato de amônio, uma banda bem definida e de alta intensidade em $1390 \mathrm{~cm}^{-1}$ indica a presença de íon amônio; e as bandas intensas em 1108 e $620 \mathrm{~cm}^{-1}$ a presença do íon sulfato ${ }^{[27,28]}$. Esses resultados estão condizentes com a incorporação dos fertilizantes visto que os hidrogéis de PAAm e MC intumescidos em água não apresentam as bandas supracitadas. Adicionalmente, mudanças significativas em ambos os espectros na região entre $1550-1830 \mathrm{~cm}^{-1[29]}$ (região características de vibrações de grupos amida) foram observadas, sendo um indício que uma das possíveis vias de interação fertilizante-matriz polimérica ocorrem com os segmentos $\mathrm{CONH}_{2}$. Uma outra possível via de interação ocorre através da interação com os grupamentos hidroxila. Porém, somente leves variações espectroscópicas em regiões acima de $3200 \mathrm{~cm}^{-1}$ foram observadas nos espectros FTIR, por isso os dados não foram apresentados. Uma possível razão é que essa região também é característica de vibrações de ligações de outros grupamentos presentes na estrutura polimérica, por exemplo, vibrações de grupamentos $\mathrm{NH}_{2}$.

\section{Parâmetros cinéticos dos hidrogéis}

Os parâmetros associados à cinética de intumescimento são de suma importância para o entendimento do mecanístico do processo de difusão solução de intumescimento para o interior do hidroge ${ }^{[13]}$. De posse de curvas de $\ln \mathrm{M}_{\mathrm{t}} \mathrm{M}_{\mathrm{eq}} v s \ln \mathrm{t}$ obtidas para hidrogéis com diferentes teores de AAm e MC e em diferentes soluções de intumescimento foi possível obter os valores de $n$ e $k$ através da Equação 1. Para hidrogéis em formato cilíndrico, os valores de $n$ entre 0,45 e 0,50 correspondem à difusão Fickiana. Um valor de $n$ aproximadamente 1,0 indica que a difusão do solvente para o interior do gel ocorre por relaxamento das cadeias que compõe a rede. Para $0,5<\mathrm{n}<1,0$ a difusão ocorre por transporte anômalo. Neste caso, o processo de difusão é realizado, ao mesmo tempo por difusão e relaxação das cadeias do hidrogel ${ }^{[16]}$.

A Tabela 1 mostra os valores obtidos para as constantes cinéticas $n$ e $k$ dos hidrogéis estudados em diferentes condições de carga iônica. O processo de intumescimento apresentado pelo hidrogel em água $\left(\mathrm{k}=0,2298 \mathrm{~h}^{-1}\right)$ foi duas vezes mais lento quando comparado ao meio $\mathrm{AlCl}_{3}\left(\mathrm{k}=0,4807 \mathrm{~h}^{-1}\right)$. O valor de $n=0,47$ demonstrou que o mecanismo de absorção do hidrogel em água corresponde à difusão Fickiana, ou seja, seu mecanismo pode ser explicado

Tabela 1. Valores de $\mathrm{Q}_{\mathrm{eq}}, k$ e $n$ obtidos para diferentes meios salinos de intumescimento utilizando o hidrogel composto por 9,0\% AAm e 1,0\% MC.

\begin{tabular}{cccc}
\hline $\begin{array}{c}\text { Meio de } \\
\text { intumescimento }\end{array}$ & $\mathbf{Q}_{\text {eq }}(\mathbf{g} / \mathbf{g})$ & $\boldsymbol{k}\left(\mathbf{h}^{-1}\right)$ & $\boldsymbol{n}$ \\
\hline $\mathrm{H}_{2} \mathrm{O}$ & $31,2 \pm 0,6$ & 0,2298 & 0,47 \\
$\mathrm{NaCl}$ & $17,3 \pm 0,2$ & 0,3166 & 0,36 \\
$\mathrm{KCl}$ & $17,3 \pm 0,1$ & 0,3230 & 0,36 \\
$\mathrm{NH}_{4} \mathrm{Cl}$ & $17,1 \pm 0,2$ & 0,3251 & 0,35 \\
$\mathrm{CaCl}_{2}$ & $16,1 \pm 0,1$ & 0,3095 & 0,34 \\
$\mathrm{MnCl}_{2}$ & $16,1 \pm 0,1$ & 0,3018 & 0,35 \\
$\mathrm{MgCl}_{2}$ & $16,7 \pm 0,1$ & 0,3093 & 0,36 \\
$\mathrm{AlCl}_{3}$ & $10,7 \pm 0,2$ & 0,4807 & 0,23 \\
\hline
\end{tabular}

em termos da $1^{\text {a }}$ e $2^{\text {a }}$ Leis de Fick, mostradas nas Equações 2 e 3 respectivamente. Assim uma substância irá se difundir mais rápido quando o gradiente de concentração for mais abrupto, ou quando o coeficiente de difusão $\left(D_{\mathrm{S}}\right)$ é aumentado. A $2^{\mathrm{a}}$ Lei de Fick se aplica na difusão não estacionária, ou seja, quando a concentração dentro do volume de difusão muda com o tempo.

$$
J_{s}=-D_{s} \frac{\Delta C_{s}}{\Delta x}
$$

onde $\mathrm{J}_{\mathrm{S}}$ representa a velocidade de transporte ou densidade de fluxo. É a quantidade de uma substância $S$ que atravessa uma unidade de área, por uma unidade de tempo. $\mathrm{D}_{\mathrm{S}}$ é o coeficiente de difusão, $\Delta \mathrm{C}_{\mathrm{S}}$ é o gradiente de concentração e $\Delta x$ é a distância.

$$
\frac{\partial C_{s}}{\partial t}=D_{s} \frac{\partial^{2} C_{s}}{\partial x^{2}}
$$

Ainda, as constantes $n$ e $k$ são inversamente proporcionais, ou seja, o aumento da carga iônica do sal no meio de intumescimento provocou diminuição de $n$ e o incremento de $k$. Estes efeitos estão relacionados com alterações na pressão osmótica do meio de intumescimento definida pela teoria de equilíbrio de Donnan ${ }^{[24]}$ e da diferença entre o gradiente de potencial químico formado entre os meios interno e externo do hidrogel ${ }^{[30]}$.

Na Tabela 2 são apresentados os valores de $n, k$ dos hidrogéis compostos por diferentes concentrações de AAm e/ou MC em diferentes soluções de intumescimento: água, soluções de sulfato de amônio e fosfato de potássio. Pode-se observar que o parâmetro $n$ se mostrou diretamente proporcional a $\mathrm{Q}_{\mathrm{eq}}$. Os valores de $k$ indicam a velocidade de difusão da solução para o interior do gel, e a mesma varia significativamente dependendo das condições de síntese dos hidrogéis e soluções de intumescimento. O valor de $k$ é aproximadamente 70 vezes maior em sulfato de amônio e em torno de 17 vezes maior em fosfato de potássio quando comparado ao hidrogel em água. Isto deve estar relacionado às interações

Tabela 2. Valores de $\mathrm{Q}_{\text {eq }}$, $k$ e $n$ obtidos para diferentes meios de intumescimento: água, soluções dos fertilizantes sulfato de amônio e fosfato de potássio.

\begin{tabular}{ccccc}
\hline \multicolumn{5}{c}{ Água } \\
\hline $\begin{array}{c}\text { Hidrogel } \\
(\mathbf{A A m} / \mathbf{M C})\end{array}$ & $\mathbf{Q}_{\mathrm{eq}}(\mathbf{g} / \mathbf{g})$ & $\boldsymbol{n}$ & $\boldsymbol{k}\left(\mathbf{s}^{-1}\right)$ & $\mathbf{R}^{2}$ \\
\hline$(6,0-1,0)$ & $49,7 \pm 3,6$ & 0,59 & 0,0018 & 0,9858 \\
$(9,0-0)$ & $16,6 \pm 0,1$ & 0,47 & 0,0050 & 0,9899 \\
$(9,0-0,5)$ & $17,7 \pm 2,1$ & 0,48 & 0,0053 & 0,9920 \\
$(9,0-1,0)$ & $25,7 \pm 1,5$ & 0,52 & 0,0037 & 0,9988 \\
$(12,0-1,0)$ & $16,9 \pm 0,1$ & 0,49 & 0,0040 & 0,9926 \\
\hline \multicolumn{5}{c}{ Sulfato de amônio } \\
\hline$(6,0-1,0)$ & $25,0 \pm 1,2$ & 0,21 & 0,10 & 0,9794 \\
$(9,0-0)$ & $13,5 \pm 0,1$ & 0,12 & 0,35 & 0,9937 \\
$(9,0-0,5)$ & $13,2 \pm 0,1$ & 0,18 & 0,17 & 0,9166 \\
$(9,0-1,0)$ & $16,7 \pm 0,2$ & 0,20 & 0,12 & 0,9517 \\
$(12,0-1,0)$ & $10,2 \pm 0,2$ & 0,16 & 0,23 & 0,9403 \\
\hline \multicolumn{5}{c}{ Fosfato de potássio } \\
\hline$(6,0-1,0)$ & $25,9 \pm 0,3$ & 0,30 & 0,020 & 0,9537 \\
$(9,0-0)$ & $13,6 \pm 0,1$ & 0,24 & 0,086 & 0,9845 \\
$(9,0-0,5)$ & $13,8 \pm 0,2$ & 0,27 & 0,041 & 0,9442 \\
$(9,0-1,0)$ & $17,5 \pm 0,1$ & 0,28 & 0,035 & 0,9142 \\
$(12,0-1,0)$ & $10,2 \pm 0,1$ & 0,26 & 0,058 & 0,9850 \\
\hline
\end{tabular}

$*[\mathrm{AAm}]=\%(\mathrm{~m} / \mathrm{v})$ e $[\mathrm{MC}]=\%(\mathrm{~m} / \mathrm{v})$. 


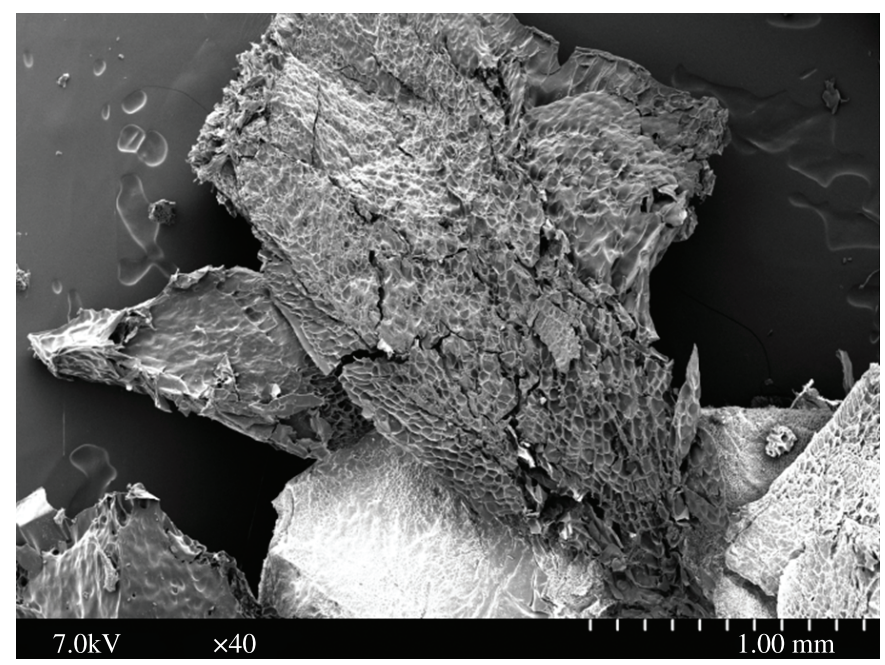

(a)

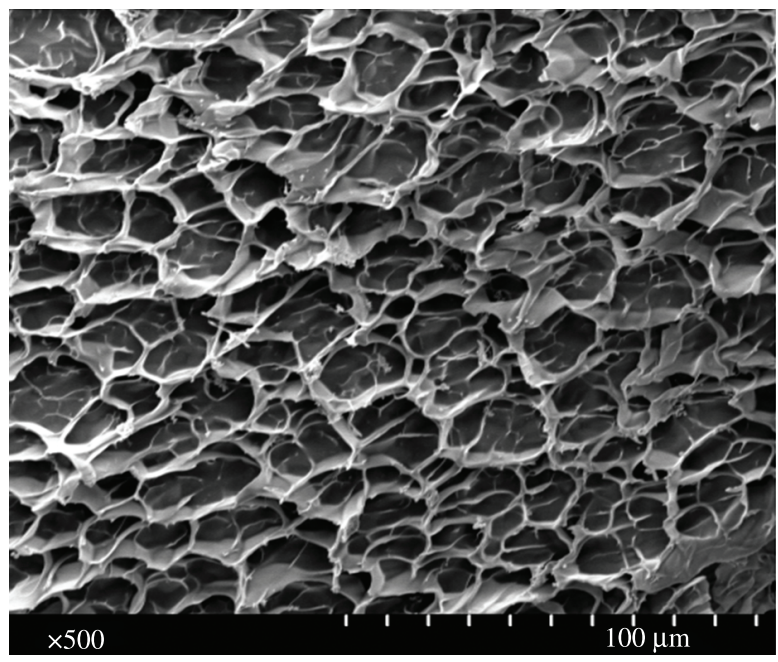

(b)

Figura 6. Micrografias, obtidas por MEV, da matriz de hidrogel composto por 6,0\% AAm e 1,0\% MC. O hidrogel foi liofilizado após ser intumescido a $25,0 \pm 0,1{ }^{\circ} \mathrm{C}$. As micrografias possuem ampliação de (a) 40 e (b) $500 \times$.

moleculares das cadeias presentes no hidrogel com a solução de intumescimento. De maneira geral, o incremento de MC provoca a diminuição da constante $k$, e o incremento de AAm aumenta $k$.

\section{Potencialidade de aplicação em sistemas de liberação controlada da matriz porosa de hidrogel}

Na Figura 6 é apresentada a morfologia obtida por microscopia eletrônica de varredura das redes tridimensionais do hidrogel de PAAm-MC, onde foi constatado que o hidrogel é extremamente poroso possuindo formas bem definidas e com estreita distribuição de tamanho de poros.

Pela alta absorção de água dos hidrogéis em um intervalo de tempo intermediário, estes materiais podem ser considerados como promissores para serem aplicados em diferentes campos na agricultura, destacando a liberação controlada de fertilizantes. Essa potencialidade de aplicação foi verificada e os resultados estão discutidos detalhadamente em um artigo recentemente publicado pelo nosso grupo de pesquisa ${ }^{[31]}$. Por exemplo, o hidrogel composto por $6,0 \%$ AAm e $1,0 \%$ MC liberou entre $80-85 \%$ de $\left(\mathrm{NH}_{4}\right)_{2} \mathrm{SO}_{4}$ e $40-45 \%$ de $\mathrm{KH}_{2} \mathrm{PO}_{4}$ da quantidade inicial sorvida; e para cada 1 grama de hidrogel seco testado observou liberação de aproximadamente $50 \mathrm{mg}$ de cada fertilizante.

\section{Conclusões}

Análises de FTIR comprovam a habilidade do hidrogel em sorver os fertilizantes fosfato de potássio e sulfato de amônio. O grau de intumescimento (hidrofilicidade) aumentou com a inserção do polissacarídeo MC nas redes tridimensionais do polímero e diminuiu à medida que a concentração de AAm foi aumentada, devido ao aumento da rigidez das cadeias poliméricas. $\mathrm{O}$ aumento da carga iônica do contra-íon do sal X-Cl e a presença de fertilizante também são fatores que diminuem consideravelmente o grau de intumescimento; devido ao decréscimo da expansão das redes tridimensionais que formam os hidrogéis ocasionado por interações dos grupamentos iônicos $\mathrm{NH}_{4}^{+}$e $\mathrm{K}^{+}$oriundos dos fertilizantes com os grupamentos hidroxila oriundo da $\mathrm{MC}$ e grupamentos $\mathrm{SO}_{4}^{2-}, \mathrm{H}_{2} \mathrm{PO}^{-}, \mathrm{PO}_{4}^{2-}$ - segmentos $\mathrm{CONH}_{2}$ presentes nas cadeias poliméricas de PAAm e MBAAm. As propriedades cinéticas dos hidrogéis puderam ser controladas pelos parâmetros estudados. A potencialidade de aplicação foi testada e os resultados indicam que o perfil de liberação é lento e sustentado para ambos os fertilizantes.
$\mathrm{O}$ hidrogel liberou até $80-85 \%$ de $\left(\mathrm{NH}_{4}\right)_{2} \mathrm{SO}_{4}$ e $40-45 \%$ de $\mathrm{KH}_{2} \mathrm{PO}_{4}$; e para cada 1 grama de hidrogel seco testado observou liberação de aproximadamente $50 \mathrm{mg}$ de cada fertilizante.

\section{Agradecimentos}

Os autores agradecem aos órgãos de fomento CAPES, CNPq e FAPESP-CMDMC, Embrapa (Programa Labex e Projeto MP1), Instituto Nacional de Ciências dos Materiais em Nanotecnologia (INCTMN) e FINEP/LNNA pelo suporte financeiro e bolsas concedidas

\section{Referências Bibliográficas}

1. Oviedo, I. R.; Mendez, N. A. N.; Gomez, M. P. G.; Rodriguez, H. C. \& Martinez, A. R. - Int. J. Polym. Mater., 57, p.1095 (2008). http://dx.doi. org/10.1080/00914030802341661

2. Aouada, F. A.; Moura, M. R.; Campese, G. M.; Girotto, E. M.; Rubira, A. F. \& Muniz, E. C.- Polímeros, 18, p.126 (2008).

3. Peppas, N. A.; Bures, P.; Leobandung, W. \& Ichikawa, H. - Eur. J. Pharm. Biopharm., 50, p.27 (2000). http://dx.doi.org/10.1016/ S0939-6411(00)00090-4

4. Leone, G.; Delfini, M.; Di Cocco, M. R.; Borioni, A. \& Barbucci, R.-Carbohydr. Res., 343, p.317 (2008).PMid:19181562 . http://dx.doi. org/10.1016/j.carres.2007.10.017

5. Sorbara, L.; Jones, L. \& Williams-Lyn, D. - Contact Lens and Anterior Eye, 32, p.93 (2009). http://dx.doi.org/10.1016/j.clae.2008.07.005

6. Zhang, L.; Rakotondradany, F.; Myles, A. J.; Fenniri, H. \& Webster, T. J. - Biomaterials, 30, p.1309 (2009). PMid:19073342 . http://dx.doi. org/10.1016/j.biomaterials.2008.11.020

7. Lentz, R. D. - Soil Sci. Soc. Am. J., 71, p.1352 (2007). http://dx.doi. org/10.2136/sssaj2005.0380

8. Raju, K. M. \& Raju, M. P. - Polym. Int., 50, p.946 (2001).

9. Lin, C. C. \& Metters, A. T. - J. Biomed. Mater. Res. A., 83, p.954 (2007). PMid:17580324 . http://dx.doi.org/10.1002/jbm.a.31282

10. Kiritoshi, Y. \& Ishihara, K. - Polymer, 45, p.7499 (2004). http://dx.doi. org/10.1016/j.polymer.2004.09.014

11. Park, J. S.; Park, J. W. \& Ruckenstein, E. - Polymer, 42, p.4271 (2001). http://dx.doi.org/10.1016/S0032-3861(00)00768-0 
12. Aouada, F. A.; Muniz, E. C.; Vaz, C. M. P. \& Mattoso, L. H. C. - Quim. Nova, 32, p.1482 (2009). http://dx.doi.org/10.1590/ S0100-40422009000600023

13. Aouada, F. A.; Pan, Z.; Orts, W. J. \& Mattoso, L. H. C. - J. Appl. Polym. Sci., 114, p.2139 (2009). http://dx.doi.org/10.1002/app.30339

14. Nilasaroya, A.; Poole-Warren, L. A.; Whitelock, J. M. \& Martens, P. J. - Biomaterials, 29, p.2008 (2008).

15. Zhang, H. T.; Bhat, R. \& Jandt, K. D. - Acta Biomater, 5, p.448 (2009).

16. Ritger, P. L. \& Peppas, N. A. - J. Control. Release, 5, p.37 (1987). http://dx.doi.org/10.1016/0168-3659(87)90035-6

17. Singh, B.; Sharma, N. \& Chauhan, N. - Carbohydr. Polym., 69, p.631 (2007). http://dx.doi.org/10.1016/j.carbpol.2007.01.020

18. Zhou, Y.; Yang, D.; Gao, X.; Chen, X.; Xu, Q.; Lu, F. \& Nie, J. - Carbohydr. Polym., 75, p.293 (2009). http://dx.doi.org/10.1016/j. carbpol.2008.07.024

19. Yu, H. \& Xiao, C. - Carbohydr. Polym., 72, p.479 (2008). http://dx.doi. org/10.1016/j.carbpol.2007.09.023

20. Flory, P. J. \& Rehner, J. J. - J. Chem. Phys., 11, p.521 (1943).

21. Aouada, F. A.; Guilherme, M. R.; Campese, G. M.; Girotto, E. M.; Rubira, A. F. \& Muniz, E. C. - Polym. Test., 25, p.158 (2006). http:// dx.doi.org/10.1016/j.polymertesting.2005.11.005

22. Aouada, F. A.; Chiou, B.-S.; Orts, W. J. \& Mattoso, L. H. C. - Polym. Eng. Sci., 49, p.2467 (2009). http://dx.doi.org/10.1002/pen.21505
23. Liang, H. F.; Hong, M. H.; Ho, R. M.; Chung, C. K.; Lin, Y. H. Chen, C. H. \& Sung, H. W. - Macromolecules, 5, p.1917 (2004).

24. Liang, R.; Yuan, H.; Xi, G. \& Zhou, Q. - Carbohydr. Polym., 77, p.181 (2009). http://dx.doi.org/10.1016/j.carbpol.2008.12.018

25. Xu, X.; Gao, B.; Yue, Q. \& Zhong, Q. - Bioresour. Technol., 102, p.5278 (2011). PMid:21094604. http://dx.doi.org/10.1016/j. biortech.2010.10.130

26. Del Nero, M.; Galindo, C.; Barillon, R.; Halter, E. \& Madé, B. - J. Control. Release, 342, p.437 (2010).

27. Blinova, N. V.; Stejskal, J.; Trchova, M.; Prokes, J. \& Omastova, M. - Eur. Polym. J., 43, p.2331 (2007) .http://dx.doi.org/10.1016/j. eurpolymj.2007.03.045

28. Chou, C. C.-K.; Huang, S.; Chen, T.; Lin, C. \& Wang, L. - Atmos. Res., 75, p.89 (2005). http://dx.doi.org/10.1016/j.atmosres.2004.12.002

29. Lynda Merlin, D. \& Sivasankar, B. - Eur. Polym. J., 45, p.165 (2009).

30. Shang, L.; Zhang, S.; Du, H. \& Venkatraman, S. S. - J. Membr. Sci., 321, p.331 (2008). http://dx.doi.org/10.1016/j.memsci.2008.05.010

31. Bortolin, A.; Aouada, F. A.; Moura, M. R.; Ribeiro, C.; Longo, E. \& Mattoso, L. H. C. - J. Appl. Polym. Sci., no prelo.

Enviado: $10 / 12 / 10$

Reenviado: 03/08/11

Aceito: $27 / 09 / 11$ 